CAPÍTULO 2

\title{
Testes Discriminativos
}

Cristina Jansen Alves, Deborah Murowaniecki Otero, Fernanda Doring Krumreich

https://doi.org/10.4322/mp.978-65-994457-0-5.c2

\section{Resumo}

Os testes discriminativos são utilizados para determinar se existem diferenças sensoriais entre as amostras. Pode-se escolher o teste a ser aplicado de acordo com o número de amostras, sendo triangular, duo-trio e comparação pareada para quando houver duas amostras em análise, e quando se tem três ou mais amostras, pode se escolher entre os testes de ordenação, comparação múltipla ou teste $A$ - não $A$. $O$ número de julgadores apresenta variação para cada teste, sendo que, no geral, testes discriminativos podem ser realizados com pequenos grupos de pessoas. Quando há um grupo de pessoas já treinadas em análise sensorial, o grupo de trabalho pode ser menor, no entanto, um grupo de pessoas sem treinamento faz com seja necessário maior número de julgadores.

Palavras-chave: amostra, ficha, julgador, teste.

\section{Introdução}

A qualidade sensorial dos alimentos é empregada para proteção do consumidor, pois ele mesmo julga a qualidade do produto através dos seus próprios sentidos. A análise sensorial utiliza o homem como instrumento de medida, e por isso deve-se escolher o método adequado e controlar as condições de aplicação do teste, para se evitar erros causados por fatores externos e psicológicos [1].

Dentre os vários testes existentes, os testes discriminativos devem ser realizados em laboratórios, onde os julgadores são colocados em cabines individualizadas com controle de fatores ambientais, e sem comunicação entre os julgadores. O local deve ter ambiente climatizado e luz adequada, pois será 
onde as fichas de avaliação sensorial serão entregues, juntamente com um copo de água e as amostras a serem analisadas [1].

O objetivo dos testes discriminativos ou também chamados teste de diferença é determinar se as amostras diferem sensorialmente entre si. Porém, este tipo de teste não identifica qual a diferença entre as amostras (ex.: cor, sabor, textura, etc.). Por serem testes analíticos podem ser utilizados para várias finalidades como, na seleção e monitoramento de equipe, na substituição de matéria-prima, na alteração no processo de produção, no controle de qualidade, desenvolvimento de novos produtos, mudança de embalagem e no estudo da vida de prateleira de produtos [2].

\section{Testes discriminativos}

Os testes discriminativos utilizados são:

- Teste duo-trio

- Teste triangular

- Teste de comparação pareada

- Teste de ordenação

- Teste de comparação múltipla/diferença de controle

- Teste A - não A

\subsection{Teste duo-trio}

O objetivo deste teste é determinar se existe uma diferença sensorial entre uma amostra e a referência (ou padrão). Para isso, primeiramente uma amostra de referência deve ser apresentada para o julgador. Em seguida são apresentadas duas amostras, uma das quais é idêntica à referência e a qual o julgador deve identificar [1].

\subsubsection{Aplicação}

As amostras podem ser apresentadas juntamente com a referência, devendo esta ser provada primeiramente, ou separadamente, sendo a amostra referência apresentada primeiro e depois que essa é analisada e retirada apresenta-se as outras duas [2].

É especialmente apropriada quando a amostra de referência $(R)$ é bem conhecida pelos provadores, ou então a amostra possui sabor forte, 
apimentado ou oleoso, já que menos provas da amostra são necessárias. Essa é uma das vantagens deste teste, pois não requer muitas avaliações quando comparado ao teste triangular. Não é necessário especificar alguma característica das amostras, e sim somente perguntar ao julgador qual é a amostra igual a R, por isso é utilizado para determinar diferenças globais entre as amostras [3].

O teste duo-trio é indicado para seleção de julgadores, selecionar amostras quando se tem um número grande, e decidir mudanças de formulação [1].

\subsubsection{Julgadores}

São recomendados no mínimo 15 julgadores, sendo que a equipe de julgadores normalmente não é treinada. Nesses casos é recomendável trocar as amostras utilizadas como $\mathrm{R}$, conforme explicação abaixo:

Se $R=A$, as combinações possíveis são: $A B$ e $B A$

Se $R=B$, as combinações possíveis são: $A B$ e $B A$

Totalizando 4 combinações de apresentação das amostras aos julgadores.

\subsubsection{Procedimento}

1. Apresentar a amostra de referência identificada como R ou P (padrão);

2. Apresentar 2 amostras codificadas (uma é idêntica à referência, a outra é diferente);

3. O provador deve identificar a amostra codificada IGUAL à referência.

\subsubsection{Análise dos resultados}

Para análise dos resultados utiliza-se teste estatístico de distribuição binomial, com probabilidade de acerto da amostra Referência de 50\%, sendo considerado estatisticamente menos eficiente quando comparado ao teste triangular.

\subsubsection{Exemplo de aplicação}

Na figura 1 pode ser observado um modelo de ficha de análise sensorial para o teste duo-trio para amostras de iogurte. 


Nome: Data: $\quad$ Amostra:
Você está recebendo uma amostra referência "R" e outras
duas codificadas de iogurte de morango. Uma amostra
codificada é igual a referência e a outra é diferente. Prove as
amostras da esquerda para a direita e assinale, entre as
amostras codificadas, aquela que é IGUAL a "R".
371
Comentários:

Figura 1. Ficha de análise sensorial para o teste duo-trio.

Resultados:

\section{Total de testes: $\mathbf{4 0}$}

\section{Total de respostas corretas: 28}

De acordo com a tabela para o Teste Duo-Trio (Anexo 1) monocaudal, para um total de 40 testes o número mínimo de respostas corretas para concluirmos que há diferença significativa à $5 \%$ de significância entre as amostras testadas é de $26(p<0,05)$. Como foram obtidas 28 , e este valor é maior que 26, podemos concluir que há diferença significativa entre as amostras ao nível de 5\% de significância.

\subsection{Teste Triangular}

O objetivo do teste triangular é verificar se existe diferença perceptível entre duas amostras através da comparação de três amostras. Este teste sensorial consiste na apresentação simultânea de três amostras codificadas, duas iguais e uma diferente, nenhuma delas identificada como amostra-padrão, o que é uma das diferenças em relação ao teste duo-trio. No procedimento do teste pode-se pedir para o julgador identificar a amostra diferente. Pode ser utilizado sempre que as amostras provoquem estímulos pouco intensos e simples [1, 3]. 


\subsubsection{Aplicação}

O teste triangular é utilizado para determinar diferenças sensoriais (inespecíficas) entre dois tratamentos ou produtos. Visa identificar se houve alterações no produto ao se modificar algum tipo de ingrediente, alteração durante etapas no processamento ou no armazenamento ou ainda se é devido a embalagem; para selecionar e monitorar julgadores com habilidade em discriminar as diferenças desejadas [1].

\subsubsection{Julgadores}

Mínimo de julgadores:12

Ideal: 20 a 40 julgadores.

\subsubsection{Procedimento}

Cada julgador recebe 3 amostras codificadas e é informado que 2 são iguais e 1 é diferente. O provador é solicitado a provar as amostras da esquerda para direita e identificar a amostra diferente.

Atenção: Neste teste existem 6 combinações diferentes de apresentação das amostras (por exemplo amostra 1= A, amostra $2=B$ ) e cada um dos modos deve ser utilizado o mesmo número de vezes.

Exemplo de combinações:

Provador $1=\mathrm{AAB}$

Provador $2=A B A$

Provador $3=\mathrm{BAA}$

Provador $4=\mathrm{BBA}$

Provador $5=\mathrm{BAB}$

Provador $6=\mathrm{ABB}$

\subsubsection{Análise dos resultados}

Obtenção de dados: Escolha forçada - o provador é obrigado a escolher uma das amostras, mesmo que não consiga identificar nenhuma diferença entre as amostras provadas.

Análise de resultados: A probabilidade de, nesta prova, um dado provador escolher ao acaso uma amostra diferente é de $33,3 \%(p=1 / 3)$. $O$ número de respostas corretas necessário para se obter uma diferença 
significativa entre as amostras a um dado nível de significância pode ser obtido através da Tabela do teste triangular.

Etapas para analisar os resultados

1ํ- Somar o número total de testes aplicados;

2-. Somar o número de respostas corretas;

3ํ- Consultar a tabela do Teste Triangular para o número mínimo de respostas corretas;

Se o número de respostas for maior ao número tabelado existe diferença significativa!

\subsubsection{Exemplo de aplicação}

$\mathrm{Na}$ Figura 2 temos uma modelo de ficha para o teste triangular.No exemplo abaixo a análise se refere a uma geleia formulada com sacarose (convencional, já utilizada pela empresa) e outra com sacarose de outro fornecedor (mais barata).

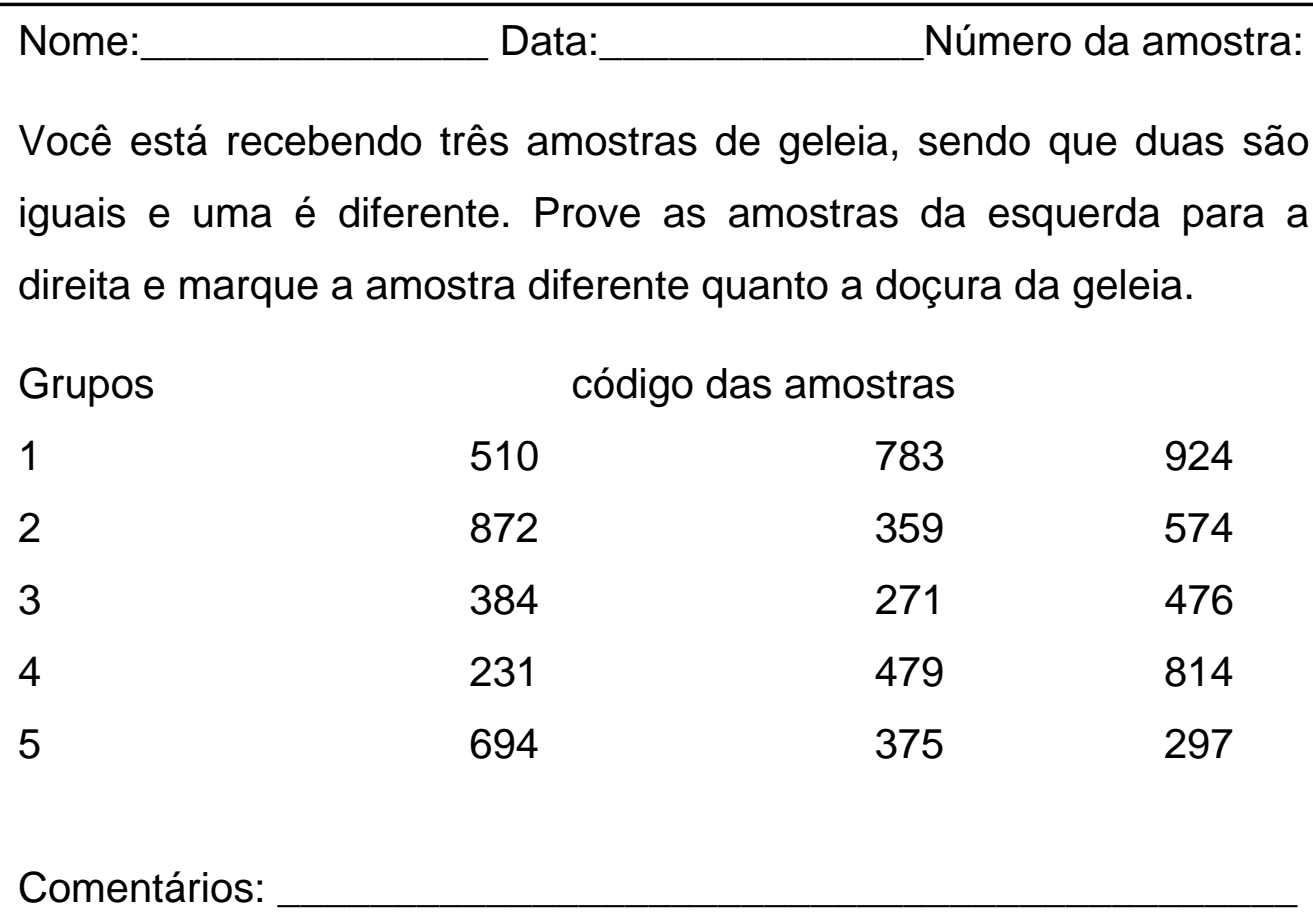

Figura 2. Ficha de análise sensorial para o teste triangular com geleias

No exemplo acima foram utilizados 12 julgadores e 5 repetições do teste $12 \times 5=60$ respostas 
É importante lembrar que, como o teste utiliza a escolha forçada de uma das amostras, é necessário fazer repetições do teste (designado por grupos na figura 2), já que existe possibilidade do julgador acertar ao acaso.

A soma do número de respostas indicando a amostra mais barata como diferente foi 19.

Então:

\section{Total de testes aplicados: 60}

\section{Número de respostas corretas: 19}

O próximo passo é ir consultar a tabela do teste triangular (Anexo 2) para $5 \%$ de significância e $n=60$

Consultando a tabela, em um total de 60 testes aplicados, o número mínimo de respostas corretas para se concluir que há diferença significativa à $5 \%$ de significância entre as amostras é de $27(p<0,05)$

27 (tabelado) é maior do que o número 19 (resultado do teste)

Portanto, não há diferença significativa entre as geleias ao nível de $5 \%$ de significância e pode-se trocar o fornecedor da sacarose, pois 0 consumidor não consegue distinguir a diferença entre as amostras.

\subsection{Teste de comparação pareada}

Os testes de comparação pareada são relativamente simples, nele avalia-se se existem diferenças entre duas amostras no que respeita à intensidade de um atributo (acidez, doçura, etc.), ou pode ser usado para determinar a preferência em relação a uma das amostras. Dada a simplicidade da prova, poderão ser utilizados julgadores com pouco treino, bastando que os mesmos estejam bem familiarizados com o atributo que se deseja testar [4].

\subsubsection{Aplicação}

O teste de comparação pareada pode ser direcional, detectando pequenas diferenças entre amostras quanto a um atributo específico ou estabelecendo a existência de uma preferência. Pode ser aplicado para selecionar e treinar julgadores. Apresenta a vantagem de facilidade de aplicação e menor fadiga. A desvantagem do teste ocorre quando o número de amostras aumenta as comparações entre as amostras [3]. 


\subsubsection{Julgadores}

Para que a análise possua uma boa representatividade, recomenda-se 0 uso de no mínimo 15 julgadores selecionados, porém com equipe altamente treinada pode-se trabalhar com 8 a 9 julgadores [5].

\subsubsection{Procedimento}

Duas amostras são apresentadas simultaneamente para comparação ou detecção de diferença em ordem balanceada ou ao acaso nas permutações $A B$ e BA. Podem ser simples (identificar se duas amostras podem ser diferenciadas, sendo mais indicado para o teste de preferência) e direcional que identifica características marcantes [3]. Cabe ao julgador identificar a amostra codificada que apresenta o atributo específico diferente ou a amostra preferida. Ao julgador deve-se fazer uma pergunta específica relevante, referindo-se à diferença, diferença direcional ou preferência. Perguntas sobre diferença e preferência não devem ser combinadas. A escolha da amostra é forçada [4].

O teste unilateral é utilizado quando a priori se sabe que existe diferença entre amostras, mas, deseja saber se esta diferença é perceptível sensorialmente. $O$ teste bilateral é empregado quando não se sabe se existe diferença entre amostras ou na avaliação da preferência [4].

O teste consiste na apresentação de duas amostras e o julgador deve apontar qual das duas tem maior intensidade com relação a um atributo específico. A probabilidade de acertos é de $50 \%(p=1 / 2)$.

\subsubsection{Análise dos resultados}

A interpretação do resultado se baseia no número de julgamentos totais versus o número de julgamentos corretos (Quadro 1). Se o número de julgamentos corretos for maior ou igual ao valor tabelado, unilateral e bilateral, conclui-se que existe diferença significativa entre as amostras ao nível de probabilidade correspondente [5]. 
Quadro 1. Modelo de casualização e resultado para comparação pareada

\begin{tabular}{|c|c|c|c|c|c|}
\hline \multicolumn{6}{|c|}{ Amostra: } \\
\hline \multicolumn{6}{|c|}{ Número de codificação: } \\
\hline Número & $\begin{array}{l}\text { Nome } \quad \text { do } \\
\text { julgador }\end{array}$ & $\begin{array}{l}\text { Ordem } \\
\text { apresenta }\end{array}$ & & $\begin{array}{l}\text { Resposta do } \\
\text { julgador }{ }^{*}(\mathrm{C})\end{array}$ & Comentário \\
\hline 1 & & $A$ & $\mathrm{~B}$ & & \\
\hline 2 & & B & $A$ & & \\
\hline 3 & & A & $\mathrm{B}$ & & \\
\hline 4 & & B & $A$ & & \\
\hline 5 & & $A$ & $\mathrm{~B}$ & & \\
\hline 6 & & B & $A$ & & \\
\hline 7 & & A & $\mathrm{B}$ & & \\
\hline$P$ & & & & & \\
\hline \multicolumn{6}{|c|}{$N$ de julgadores totais $(p)$} \\
\hline \multicolumn{6}{|c|}{$\mathrm{N}$ de julgadores corretos } \\
\hline $\begin{array}{l}\text { Valor to } \\
\text { probabili }\end{array}$ & $\begin{array}{l}\text { belado (nível de } \\
\text { lade) }\end{array}$ & & & & \\
\hline
\end{tabular}

${ }^{*}$ Correta (C) Errada (E) $\quad \mathrm{p}=\mathrm{n}^{\circ}=$ de julgadores ou julgamentos

\subsubsection{Exemplo de aplicação}

1) O proprietário de uma empresa de sucos deseja saber se ao aumentar a acidez titulável de seu suco em $0,1 \%$ aumentará também a acidez percebida sensorialmente. Assim sendo, ele aplicou um teste de comparação de acordo com a ficha abaixo (Figura 3): 


\section{Ficha de avaliação}

Nome:

Data:

Instruções: Você está recebendo duas amostras de suco de maracujá com diferentes concentrações de acidez. Prove as amostras codificadas da esquerda para a direita e assinale o código da amostra mais ácida.

\begin{tabular}{|l|l|l|}
\hline Código das amostras & Amostra mais ácida & Comentário \\
\hline 572 & & \\
\hline 647 & & \\
\hline
\end{tabular}

Figura 3. Modelo de ficha de avaliação para teste de comparação pareada

2) Uma grande fábrica de biscoitos necessitou substituir seu fornecedor de polvilho. Sabendo que a matéria prima apresenta influência sob as características finais do produto, o químico de alimentos responsável pela produção optou por aplicar um teste comparativo entre os dois novos fornecedores, visando verificar se essas novas matérias primas irão produzir biscoitos com a mesma dureza e para tal, usou a ficha de avaliação abaixo (Figura 4).

\section{Ficha de avaliação}

Nome:

Data:

Instruções: Você está recebendo duas amostras codificadas de biscoito de polvilho. Por favor, avalie as amostras da esquerda para a direita quanto à dureza e assinale o código referente à amostra mais dura.

261

843

Comentários:

Figura 4. Apresentação de modelo de ficha para análise de comparação aos pares. 


\subsection{Teste de ordenação}

São apresentados, em ordem aleatória, ao julgador um conjunto de mais de duas amostras. Ao provador é solicitado que as ordene de acordo com a intensidade de um determinado atributo. $O$ teste de ordenação avalia três ou mais amostras, simultaneamente, onde o juiz deve ordená-las de acordo com a intensidade de um atributo específico [6].

\subsubsection{Aplicação}

Este método deverá ser utilizado quando o objetivo é a comparação de diversas amostras no que diz respeito a um único atributo como doçura, frescor ou preferência. A ordenação é o modo mais fácil de efetuar esta comparação.

Deve-se ter atenção que os dados obtidos são meramente ordinais, não se obtendo uma medida do grau de diferença na intensidade do atributo, para que seja possível indicar se existe diferença entre as amostras é necessário aplicar análise estatística. Duas amostras consecutivas, quer difiram muito quer pouco num dado atributo, serão sempre separadas por uma unidade.

A prova de ordenação é mais rápida que outros métodos, sendo particularmente útil nos casos em que se pretende efetuar uma pré-seleção de amostras para análises subsequentes. Este método poderá também ser usado para o treino e seleção de provadores [3].

\subsubsection{Julgadores}

Dada a simplicidade da prova, esta pode ser realizada com julgadores com um treino reduzido, em geral basta que os julgadores estejam bem familiarizados com o atributo em estudo. No caso do teste se revestir de uma importância especial (por exemplo um sabor estranho num produto que já tenha sido expedido da fábrica), poderão de ter de ser usados provadores bem treinados e que tenham demonstrado uma boa acuidade para a detecção de pequenas diferenças no atributo. A prova deve ser realizada com um mínimo de 5 a 8 provadores, sendo a discriminação bastante melhorada se poderem ser utilizados 16 ou mais provadores [5].

O número de julgadores deve ser no mínimo de 5 especialistas ou 15 julgadores selecionados. Para o teste de preferência em laboratório, utilizam-se 
30 ou mais julgadores e, para o teste de consumidor, 100 ou mais. As amostras devem ser apresentadas de forma balanceada ou casualizada $[5,6]$.

\subsubsection{Procedimento}

Este teste pode ser aplicado para pré-seleção entre grande número de amostras. Uma série de três ou mais amostras codificadas aleatorizadas é apresentada ao julgador para que ordene em ordem crescente ou decrescente da intensidade do atributo específico.

Apresentam-se aos provadores um conjunto de $k$ amostras ordenadas aleatoriamente (preferencialmente segundo um desenho aleatório equilibrado) sendo-Ihes pedido que as ordenem de acordo com o atributo em questão. As amostras serão apresentadas simultaneamente, se possível, ou então sequencialmente.

O conjunto de amostras poderá ser apresentado uma só vez a cada provador ou então várias vezes, mas com codificações diferentes. A exatidão é melhorada se for possível apresentar o conjunto mais do que uma vez.

Em testes de preferência os provadores deverão ser instruídos a dar ordem 1 à amostra preferida, ordem 2 à que preferem em segundo lugar e assim sucessivamente. Em testes de intensidade (discriminativos) os provadores deverão dar ordem 1 à amostra menos intensa, 2 à amostra seguinte e $\mathrm{k}$ à amostra mais intensa [3].

Os provadores deverão começar por ordenar as amostras segundo uma classificação provisória e em seguida verificar a ordenação e proceder às alterações necessárias. Deverão ser informados que mesmo em caso de duas ou mais amostras parecerem iguais quanto ao atributo em questão deverão ordenar as amostras, no entanto e caso não as consigam ordenar, deverão indicar quais as amostras que consideraram idênticas na folha de respostas.

No caso de se pretender avaliar mais do que um atributo, as avaliações deverão ser feitas em provas independentes e utilizando uma codificação diferente de modo a que uma avaliação não influencie as outras. Poderão ser fornecidas informações específicas sobre o modo de efetuar a prova (agite antes de cheirar). No caso de o julgador degustar o produto poderá ser necessário fornecer água, pão ou outro produto para neutralizar as sensações entre as amostras [5]. 


\subsubsection{Análise dos resultados}

O resultado é dado pela soma das ordens obtidas dos julgadores a cada uma das amostras. A avaliação dos resultados é feita pelo teste de Friedman utilizando a tabela de Newell e MacFarlane (Anexo 3 e 4) para verificar se há ou não diferença significativa entre as amostras. Se a diferença entre as somas das ordens for maior ou igual ao valor tabelado, conclui-se que existe diferença significativa entre as amostras ao nível de significância correspondente [3]. A avaliação dos resultados obtidos através do teste de ordenação para cada amostra pode ser tabulado conforme ficha exemplificada no Quadro 2.

Quadro 2. Modelo de casualização e tabulação dos resultados do teste de ordenação.

\begin{tabular}{|c|c|c|c|c|c|c|}
\hline \multicolumn{2}{|c|}{$\begin{array}{l}\text { Amostra: } \\
\text { Número de codificação: } \quad(A)\end{array}$} & \multicolumn{2}{|c|}{$(\mathrm{B})$} & $(\mathrm{C})$ & & \multirow[b]{2}{*}{ Comentários } \\
\hline Número & Nome do julgador & \multicolumn{4}{|c|}{ Ordem de apresentação } & \\
\hline 1 & & $A$ & B & $\mathrm{C}$ & $\mathrm{D}$ & \\
\hline 2 & & $A$ & $\mathrm{C}$ & $\mathrm{B}$ & $\mathrm{D}$ & \\
\hline 3 & & $B$ & $A$ & $\mathrm{D}$ & $\mathrm{C}$ & \\
\hline 4 & & $\mathrm{~B}$ & C & $A$ & $\mathrm{D}$ & \\
\hline 5 & & & $\mathrm{D}$ & $\mathrm{B}$ & $A$ & \\
\hline 6 & & $\mathrm{C}$ & $A$ & $\mathrm{D}$ & B & \\
\hline 7 & & $\mathrm{D}$ & $\mathrm{B}$ & $A$ & $\mathrm{C}$ & \\
\hline \multicolumn{7}{|l|}{$\mathrm{P}$} \\
\hline \multicolumn{2}{|c|}{ Tipos de amostras ou tratamentos } & $(A)$ & (B) & $(\mathrm{C})$ & (D) & \\
\hline \multicolumn{2}{|c|}{ Soma das ordens } & $\begin{array}{l}\sum(A) \\
\sum(B)\end{array}$ & & $\Sigma(\mathrm{C})$ & $\sum(\mathrm{D})$ & \\
\hline \multicolumn{2}{|c|}{$\mathrm{N}$ de julgamentos $(\mathrm{p})$} & & & & & \\
\hline \multicolumn{2}{|c|}{$\mathrm{N}$ de amostras ou tratamentos $(\mathrm{t})$} & & & & & \\
\hline \multicolumn{2}{|c|}{$\begin{array}{l}\text { Valor tabelado (nível de } \\
\text { significância) }\end{array}$} & & & & & \\
\hline
\end{tabular}


Teste de Friedman - Com o número de amostras ou tratamentos avaliados (t) e o número de julgamentos ( $p$ ) obtidos, utiliza-se a tabela de Newel e MacFarlane, para obter a diferença crítica entre os totais de ordenação. Se as diferenças entre as somas das ordens de duas amostras (Quadro 3) diferirem por um valor maior ou igual ao valor tabelado (crítico), existe diferença significativa entre elas ao nível testado [4].

Quadro 3. Módulos de diferenças entre as somas das ordens das amostras

\begin{tabular}{|l|l|l|l|l|}
\hline \multicolumn{1}{|c|}{ Amostras } & \multicolumn{1}{|c|}{$(\mathrm{A})$} & \multicolumn{1}{|c|}{$(\mathrm{B})$} & \multicolumn{1}{c|}{$(\mathrm{C})$} & \multicolumn{1}{c|}{$(\mathrm{D})$} \\
\hline Somatório total & $\Sigma(\mathrm{A})$ & $\Sigma(\mathrm{B})$ & $\Sigma(\mathrm{C})$ & $\Sigma(\mathrm{D})$ \\
\hline Diferença versus A & & $\Sigma(\mathrm{A})-\Sigma(\mathrm{B})$ & $\Sigma(\mathrm{A})-\Sigma(\mathrm{C})$ & $\Sigma(\mathrm{A})-\Sigma(\mathrm{D})$ \\
\hline Diferença versus B & & & $\Sigma(\mathrm{B})-\Sigma(\mathrm{C})$ & $\Sigma(\mathrm{B})-\Sigma(\mathrm{D})$ \\
\hline Diferença versus C & & & & $\Sigma(\mathrm{C})-\Sigma(\mathrm{D})$ \\
\hline
\end{tabular}

\subsubsection{Exemplo de aplicação}

1) Uma indústria de alimentos deseja diminuir a concentração de açúcar usado na formulação de suas geleias visando atender a um novo nicho de mercado. Para isso alguns testes foram realizados e quatro novas formulações foram selecionadas, as quais utilizam concentrações muito próximas de açúcar. Visando avaliar a percepção dos consumidores quanto ao atributo em estudo, um teste de ordenação foi realizado conforme ficha abaixo (Figura 5).

\section{Ficha de avaliação}

Nome:

Data:

Instrucões: Você está recebendo quatro amostras codificadas de geleias. Por favor avalie as amostras da esquerda para a direita e ordene-as quanto ao atributo doçura em ordem crescente.

1.

2.

3.

4.

Comentários:

Figura 5. Ficha para aplicação de teste de ordenação. 


\subsection{Teste de comparação múltipla ou diferença de controle}

O teste de comparação múltipla ou diferença de controle, como também pode ser denominado é aplicado quando se quer determinar se existe diferença entre uma amostra padrão e uma ou mais amostras-teste, bem como estimar o grau de diferença existente. Este teste é indicado para avaliação de apenas um único atributo sensorial. Por exemplo, faz-se a pergunta se a amostra apresentada é pior ou melhor que a amostra padrão, através do grau de diferença que poderá ser uma escala verbal, numérica ou mista [5].

Logo, o teste tem como princípio apresentar uma amostra controle, mais uma ou mais amostras codificadas, solicitando que o julgador avalie cada amostra em relação ao controle segundo determinado atributo [5].

\subsubsection{Aplicação}

Este teste pode ser utilizado em desenvolvimento e melhoramento de produtos, como: substituição de ingredientes ou de materiais de embalagens, bem como em mudanças nos processos ou de condições e tempo de estocagem, controle de qualidade e estabilidade de armazenamento [3].

Esse teste tem como vantagem ser uma técnica adequada para as discriminações visuais em que a fadiga sensorial pode ser menos frequente e a quantidade da amostra para o teste não é necessariamente grande, podendo 0 mesmo grupo de amostras ser avaliado por toda a equipe de julgadores. Nesse sentido, é eficiente para análise de 4 a 5 amostras simultâneas, quando se necessita de uma medida de magnitude (tamanho) e direção da diferença e também, eficientes para detectar pequenas diferenças de amostras. Enquanto como desvantagem, podemos citar a fadiga para um número grande de amostras [3].

\subsubsection{Julgadores}

A equipe deve ser de 20 a 50 julgadores pré-selecionados, sendo que 0 número de julgadores deve ser no mínimo 7 especialistas ou 15 treinados.

\subsubsection{Procedimento}

Uma amostra padrão $(P)$ e uma ou mais amostras codificadas são entregues ao julgador, sendo-lhe solicitado que assinale o grau de diferença 
entre cada amostra e o padrão segundo sua percepção em relação a um determinado atributo.

\subsubsection{Análise dos resultados}

Os resultados são discutidos por meio de análise estatística, utilizando teste de análise de variância e comparação múltipla de médias. Deve-se utilizar o teste de Dunnett unilateral quando existe diferença entre amostras e bilateral quando não se sabe se existe diferença.

\subsubsection{Exemplo de aplicação}

Uma indústria de alimentos deseja avaliar a crocância de suas batatas chips. Para isso um teste de comparação múltipla ou diferença de controle foi realizado, onde uma amostra padrão $(P)$ e três amostras codificadas foram apresentadas aos julgadores, os quais deveriam avaliar as amostras quanto à crocância, assinalando o grau de diferença entre cada amostra e o padrão segundo sua percepção, conforme ficha abaixo (Figura 6).

TESTE DE COMPARAÇÃO MÚLTIPLA OU DIFERENÇA DE CONTROLE

Nome: Data:

Você receberá uma amostra padrão $(P)$ e três amostras codificadas. Por favor, avalie as amostras da esquerda para direita quanto à crocância e assinale o grau de diferença entre cada amostra e o padrão anotando o código da amostra correspondente à escala segundo sua percepção.

Código da amostra:

1. ( ) Extremamente mais crocante que $P$

2. ( ) Muito mais crocante que P

3. ( ) Moderadamente mais crocante que $P$

4. ( ) Ligeiramente mais crocante que $P$

5. ( ) Não há diferença entre $P$ e a amostra quanto à crocância

6. ( ) Ligeiramente menos crocante que $P$

7. ( ) Moderadamente menos crocante que $P$

8. () Muito menos crocante que $P$

9. ( ) Extremamente menos crocante que $P$

Comentários:

Figura 6. Ficha de análise sensorial para o teste comparação múltipla ou diferença de controle. 


\subsection{Teste "A" ou "Não A"}

O teste "A" ou "Não A" é aplicado para avaliar amostras que apresentem variações de aparência ou de gosto remanescente, ou seja, este teste é usado quando os testes duo-trio e triangular não são aplicáveis. Uma das vantagens da utilização deste teste é admitir pequenas diferenças no mesmo tipo de amostra, enquanto a desvantagem cita-se em relação à fadiga sensorial [7].

\subsubsection{Aplicação}

O teste é aplicado quando, por exemplo, queremos determinar diferenças entre produtos resultantes de alterações em ingredientes, processo, embalagem ou armazenamento, bem como para determinar se existe diferença global quando atributo(s) específico(s) não pode(m) ser identificados como tendo sido afetados é que este teste se mostra efetivo [8].

O teste " $A$ " ou "Não $A$ " tem como princípio uma série de amostras provenientes de dois produtos ("A" ou "Não A" é apresentada para identificação das amostras "A"). Assim podemos determinar a habilidade em discriminar comparando as identificações corretas com as incorretas, usando o teste quiquadrado [8].

\subsubsection{Julgadores}

Para a utilização deste teste, necessita-se treinar de 10 a 50 julgadores com as amostras denominadas "A" e "Não A" utilizando de 20 a 50 apresentações de cada amostra em estudo. Em relação a cada provador, este por sua vez pode receber somente uma amostra ("A" e "Não $A$ "), duas amostras (uma "A" e uma "Não A“), ou uma série de até 10 amostras. $O$ número de amostras permitido no teste é determinado pelo grau de fadiga física e/ou mental que produz nos provadores.

\subsubsection{Procedimento}

Para a realização do teste as amostras devem ser fornecidas de forma aleatória, podendo ser "A" ou "Não A", e neste caso, pede-se para que a amostra "A" seja identificada (Figura 7). 


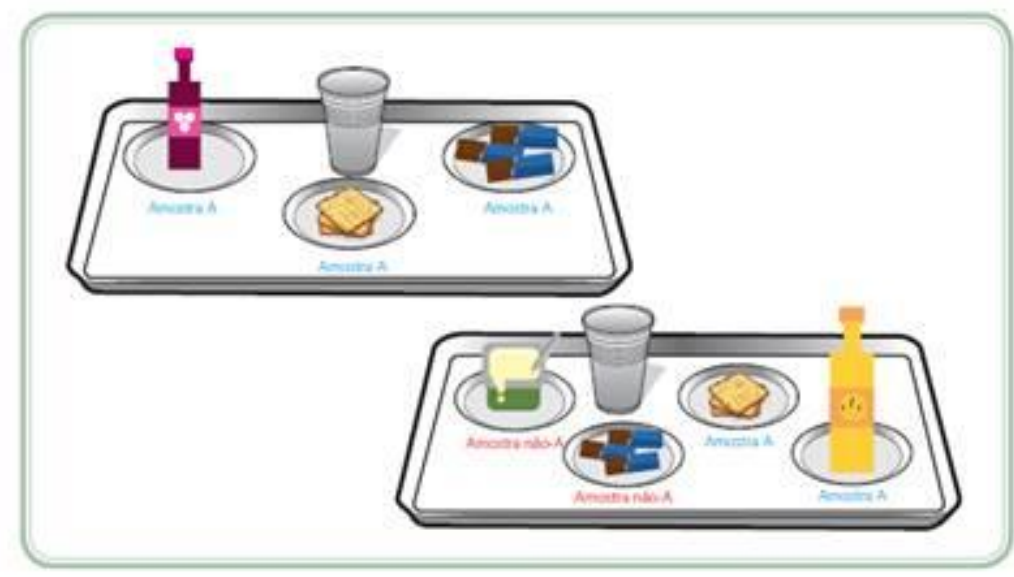

Figura 7. Apresentação da amostra "A" várias vezes, para o julgador poder identificar a amostra "A".

\subsubsection{Análise dos resultados}

Os resultados são analisados conforme a tabela de valores críticos do qui-quadrado (Anexo 5).

\subsubsection{Exemplo de aplicação}

Um estudante da área de alimentos está pesquisando adoçantes alternativos para um néctar de pêssego que contém $6 \%$ de açúcar na sua formulação. Testes preliminares de gosto estabeleceram que o uso de $0,2 \%$ do novo adoçante é equivalente a $5 \%$ de sacarose. Sendo assim, o estudante deseja saber se as duas bebidas (usando 6\% de açúcar e 0,2\% do novo adoçante) se distinguem pelo sabor. Vinte julgadores recebem de forma aleatória cinco amostras "A" (néctar com adoçante) e cinco amostras "Não A" (néctar com sacarose), sendo-lhes recomendado que determinem quais amostras são "A", conforme ficha abaixo (Figura 8). 


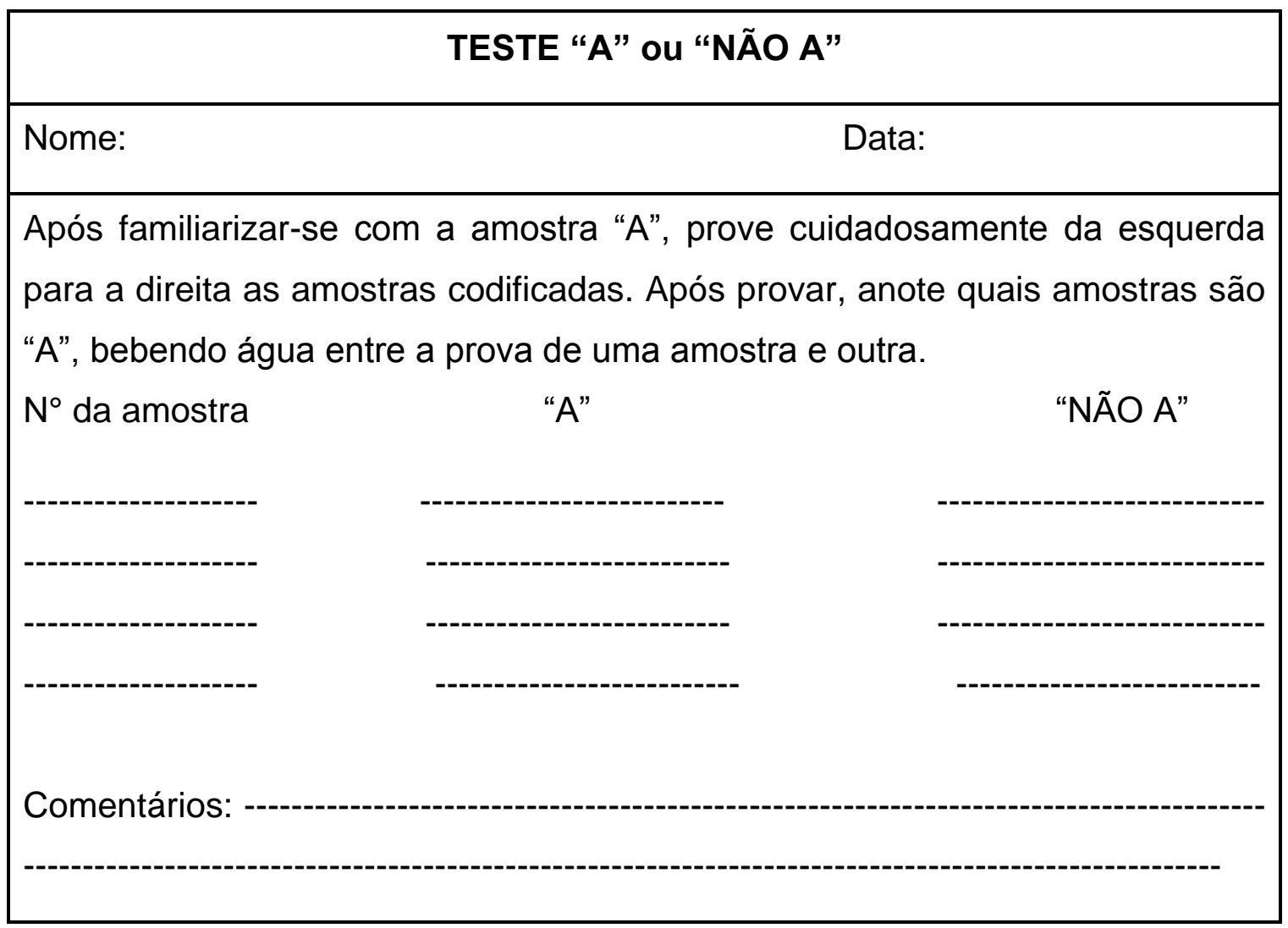

Figura 8. Modelo de ficha sensorial para aplicação do teste "A" ou "Não A".

Após aplicação do teste "A" ou "Não A", os resultados obtidos de 370 avaliações são apresentados na Tabela 1, abaixo.

Tabela 1. Julgamentos obtidos no teste "A" ou "NÃO A" de néctares.

\begin{tabular}{c|cc|c}
\hline \multirow{2}{*}{ Respostas } & \multicolumn{2}{|c|}{ Amostras } & \multirow{2}{*}{ Total } \\
\cline { 2 - 3 } & "A" & "NÃO A" & \\
\hline "A" & 115 & 113 & 228 \\
"NÃO A" & 55 & 87 & 142 \\
\hline Total & 170 & 200 & 370 \\
\hline
\end{tabular}


A construção da Tabela 1 (valores críticos do qui-quadrado) é baseada nas frequências observadas. Ela é escrita de maneira literal como mostrado na Tabela 2, abaixo, onde $\mathrm{N}$ é o total de julgamentos realizados.

Tabela 2. Valores literais de uma tabela $2 \times 2$

\begin{tabular}{|c|c|c|c|}
\hline \multirow[t]{2}{*}{ Variável X } & \multicolumn{2}{|c|}{ Variável Y } & \multirow[t]{2}{*}{ Total } \\
\hline & 1 & 2 & \\
\hline 1 & $A$ & B & $A+B$ \\
\hline 2 & $C$ & $\mathrm{D}$ & $C+D$ \\
\hline Total & $A+C$ & $B+D$ & $\mathrm{~N}$ \\
\hline
\end{tabular}

Para analisar os resultados aplica-se a distribuição qui-quadrada o qual serve para testar a hipótese de que duas variáveis categorizadas. Assim sendo, quando aplicarmos a distribuição qui-quadrada, teremos:

Frequência esperada para "A": $\frac{170 \times 228}{370}=104,76$

Frequência esperada para "NÃO A": $\frac{200 \times 142}{370}=76,76$

$$
X^{2}=\frac{115-104,76^{2}}{104,76}+\frac{113-104,76^{2}}{104,76}+\frac{55-76,76^{2}}{76,76}+\frac{87-76,76^{2}}{76,76}=9,19
$$

No anexo 5, onde constam os valores críticos do qui-quadrado, podemos observar que para o GL (grau de liberdade) 1, a nível de 5\% de significância, o valor é $X^{2}=3,84$. Assim, podemos concluir que existe diferença significativa entre os néctares adoçados com açúcar e adoçante, tendo em vista que o valor encontrado na análise estatística que foi de 9,19 é maior do que o tabelado $(3,84)$. 
Atenção: para aplicar o teste qui-quadrado são necessárias as seguintes exigências:

a) Independência: os dois aspectos das duas variáveis em comparação devem ser independentes;

b) Os julgamentos devem ser de tamanho maior do que 20.

\subsection{Considerações finais}

Os testes sensoriais discriminativos são considerados métodos objetivos utilizados em análise sensorial de alimentos, bebidas e água, com os efeitos das opiniões dos indivíduos minimizados. Visa avaliar efeitos específicos por meio de discriminação simples, indicando por comparações, se existem ou não diferenças estatísticas entre amostras.

É importante ter cuidados na padronização do preparo e apresentação das amostras e na formação da equipe sensorial. Todas as amostras devem ser codificadas com números aleatórios de três dígitos, casualizadas e apresentadas à equipe pré-selecionada e/ou treinada. Além disso, os testes devem ser conduzidos em cabines individualizadas com controle das condições ambientais, tais como: iluminação, temperatura, ausência de sons ou ruídos e livre de odores estranhos. Esses métodos são aplicáveis tanto para fins de controle de qualidade quanto para fins de pesquisa e desenvolvimento de novos produtos, quando se visa estabelecer o possível efeito de novos ingredientes ou de diferentes processos sobre as características sensoriais do produto.

\section{Referências}

[1] Palermo J. R. Análise sensorial: fundamentos e métodos. 1 ed. Rio de Janeiro: Atheneu; 2015. 170p.

[2] Teixeira L. V. Análise sensorial na indústria de alimentos. Revista do Instituto de Laticínios Cândido Tostes 2009; 366(64) https://revistadoilct.com.br/rilct/article/view/70/76\# (acessado 08 fevereiro de 2021).

[3] Gularte M.A. Manual de análise sensorial de alimentos. Pelotas: UFPel; 2009. 106p. 
[4] Carmo J.L.D. (2018). Manual de boas práticas em análise sensorial (Tese de doutorado). 112p

[5] Instituto Adolfo Lutz, I. A. L. (2008). Métodos físico-químicos para análise de alimentos. IV Edição, $1^{\circ}$ Edição digital. 533p

[6] Esteves, E. (2009). Análise sensorial. Apontamentos para as aulas teóricas de Análise Sensorial do Curso de Engenharia Alimentar. Universidade do Algarve-Instituto Superior de Engenharia. 60p

[7] Dutcosky, S. D. (2007). Análise sensorial de alimentos. 2. ed. rev. e ampl. Curitiba: Champagnat, 239p.

[8] Meilgaard, M.; Civille, G.V.; Carr, B. T. (1991). Sensory Evaluation Techniques. 2 ed. Flórida - USA: CRC Press. 354p.

[9] Associação Brasileira de Normas e Técnicas (1993). NBR 12994: métodos de análise sensorial dos alimentos e bebidas - classificação. Rio de Janeiro: ABNT.

NBR 13170: teste de ordenação em análise sensorial. Rio de Janeiro: ABNT, 1994. 
Anexo 1.Tabela de significância para cálculo do resultado do teste duo-trio

Níveis de probabilidade

\begin{tabular}{|c|c|c|c|}
\hline \multirow{2}{*}{$\begin{array}{l}\text { Número de } \\
\text { julgadores }\end{array}$} & \\
\hline & $5 \%$ & $1 \%$ & $0,1 \%$ \\
\hline 5 & 5 & - & - \\
\hline 6 & 6 & - & - \\
\hline 7 & 7 & 7 & - \\
\hline 8 & 7 & 8 & - \\
\hline 9 & 8 & 9 & - \\
\hline 10 & 9 & 10 & 10 \\
\hline 11 & 9 & 10 & 11 \\
\hline 12 & 10 & 11 & 12 \\
\hline 13 & 10 & 12 & 13 \\
\hline 14 & 11 & 12 & 13 \\
\hline 15 & 12 & 13 & 14 \\
\hline 16 & 12 & 14 & 15 \\
\hline 17 & 13 & 14 & 16 \\
\hline 18 & 13 & 15 & 16 \\
\hline 19 & 14 & 15 & 17 \\
\hline 20 & 15 & 16 & 18 \\
\hline 21 & 15 & 17 & 18 \\
\hline 22 & 16 & 17 & 19 \\
\hline 23 & 16 & 18 & 20 \\
\hline 24 & 17 & 19 & 20 \\
\hline 25 & 18 & 19 & 21 \\
\hline 26 & 18 & 20 & 22 \\
\hline 27 & 19 & 20 & 22 \\
\hline 28 & 19 & 21 & 23 \\
\hline 29 & 20 & 22 & 24 \\
\hline
\end{tabular}




\begin{tabular}{|c|c|c|c|}
\hline 30 & 20 & 22 & 24 \\
\hline 31 & 21 & 23 & 25 \\
\hline 32 & 22 & 24 & 26 \\
\hline 33 & 22 & 24 & 26 \\
\hline 34 & 23 & 25 & 27 \\
\hline 35 & 23 & 25 & 27 \\
\hline 36 & 24 & 26 & 28 \\
\hline 37 & 24 & 26 & 29 \\
\hline 38 & 25 & 27 & 29 \\
\hline 39 & 26 & 28 & 30 \\
\hline 40 & 26 & 28 & 30 \\
\hline 41 & 27 & 29 & 31 \\
\hline 42 & 27 & 29 & 32 \\
\hline 43 & 28 & 30 & 32 \\
\hline 44 & 28 & 31 & 33 \\
\hline 45 & 29 & 31 & 34 \\
\hline 46 & 30 & 32 & 34 \\
\hline 47 & 30 & 32 & 35 \\
\hline 48 & 31 & 33 & 36 \\
\hline 49 & 31 & 34 & 36 \\
\hline 50 & 32 & 34 & 37 \\
\hline 60 & 37 & 40 & 43 \\
\hline 70 & 43 & 46 & 49 \\
\hline 80 & 48 & 51 & 55 \\
\hline 90 & 54 & 57 & 61 \\
\hline 100 & 59 & 63 & 66 \\
\hline
\end{tabular}

Fonte: IAL, 2008. 
Anexo 2.Tabela de significância para cálculo do resultado do teste triangular

Níveis de probabilidade

Número de julgamentos

0,05

0,01

5

4

5

6

5

6

7

5

6

8

6

7

9

6

7

10

7

8

11

7

8

12

8

9

13

8

9

14

9

10

15

9

10

16

9

11

17

10

11

18

10

12

19

11

12

20

11

13

21

12

13

22

12

14

23

12

14

24

13

15

25

13

15

26

14

15

27

14

16 


\begin{tabular}{|c|c|c|}
\hline 28 & 15 & 16 \\
\hline 29 & 15 & 17 \\
\hline 30 & 15 & 17 \\
\hline 31 & 16 & 18 \\
\hline 32 & 16 & 18 \\
\hline 33 & 17 & 18 \\
\hline 34 & 17 & 19 \\
\hline 35 & 17 & 19 \\
\hline 36 & 18 & 20 \\
\hline 37 & 18 & 20 \\
\hline 38 & 19 & 21 \\
\hline 39 & 19 & 21 \\
\hline 40 & 19 & 21 \\
\hline 41 & 20 & 22 \\
\hline 42 & 20 & 22 \\
\hline 43 & 20 & 23 \\
\hline 44 & 21 & 23 \\
\hline 45 & 21 & 24 \\
\hline 46 & 22 & 24 \\
\hline 47 & 22 & 24 \\
\hline 48 & 22 & 25 \\
\hline 49 & 23 & 25 \\
\hline 50 & 23 & 26 \\
\hline 55 & 25 & 27 \\
\hline 60 & 27 & 30 \\
\hline 65 & 28 & 31 \\
\hline 70 & 31 & 34 \\
\hline 75 & 32 & 35 \\
\hline 80 & 35 & 38 \\
\hline 85 & 36 & 39 \\
\hline
\end{tabular}




\begin{tabular}{ccc}
\hline 90 & 38 & 42 \\
95 & 40 & 43 \\
100 & 42 & 45 \\
\hline
\end{tabular}

Fonte: ABNT, 1993.

Anexo 3. Tabela com os valores críticos para comparação com os módulos das diferenças entre as so-mas das ordens do teste de ordenação, a 5\% de significância

\begin{tabular}{|c|c|c|c|c|c|c|c|c|c|c|}
\hline \multirow{2}{*}{$\begin{array}{c}\text { № de } \\
\text { Julgamentos }\end{array}$} & \multicolumn{10}{|c|}{$\mathrm{n}^{\circ}$ de amostras ou tratamentos } \\
\hline & 3 & 4 & 5 & & & 8 & 9 & 10 & 11 & 12 \\
\hline 6 & 9 & 12 & 15 & 19 & 22 & 26 & 30 & 34 & 37 & $4 c$ \\
\hline 7 & 10 & 13 & 17 & 20 & 24 & 28 & 32 & 36 & 40 & 44 \\
\hline 8 & 10 & 14 & 18 & 22 & 26 & 30 & 34 & 38 & 43 & 47 \\
\hline 9 & 10 & 15 & 19 & 23 & 27 & 32 & 36 & 41 & 46 & 50 \\
\hline 10 & 11 & 15 & 20 & 24 & 29 & 34 & 38 & 43 & 48 & . \\
\hline 11 & 11 & 16 & 21 & 25 & 30 & 35 & 40 & 45 & 51 & 56 \\
\hline 12 & 12 & 17 & 22 & 27 & 32 & 37 & 42 & 48 & 53 & 58 \\
\hline 13 & 12 & 18 & 23 & 28 & 33 & 39 & 44 & 50 & 55 & 61 \\
\hline 14 & 13 & 18 & 24 & 29 & 34 & 40 & 46 & 52 & 57 & 63 \\
\hline 15 & 13 & 19 & 24 & 30 & 36 & 42 & 47 & 53 & 59 & 66 \\
\hline 16 & 14 & 19 & 25 & 31 & 37 & 42 & 49 & 55 & 61 & 67 \\
\hline 17 & 14 & 20 & 26 & 32 & 38 & 44 & 50 & 56 & 63 & 69 \\
\hline 18 & 15 & 20 & 26 & 32 & 39 & 45 & 51 & 59 & 65 & 71 \\
\hline 19 & 15 & 21 & 27 & 33 & 40 & 46 & 53 & 60 & 66 & 73 \\
\hline 20 & 15 & 21 & 28 & 34 & 41 & 47 & 54 & 61 & 68 & 75 \\
\hline 21 & 16 & 22 & 28 & 35 & 42 & 49 & 56 & 63 & 70 & 77 \\
\hline 22 & 16 & 22 & 29 & 36 & 43 & 50 & 57 & 64 & 71 & 79 \\
\hline 23 & 16 & 23 & 30 & 37 & 44 & 51 & 58 & 65 & 73 & 80 \\
\hline 24 & 17 & 23 & 30 & 37 & 45 & 52 & 59 & 67 & 74 & 82 \\
\hline 25 & 17 & 24 & 31 & 38 & 46 & 53 & 61 & 68 & 76 & 84 \\
\hline 26 & 17 & 24 & 32 & 39 & 46 & 54 & 62 & 70 & 77 & 85 \\
\hline 27 & 18 & 25 & 32 & 40 & 47 & 55 & 63 & 71 & 79 & 87 \\
\hline 28 & 18 & 25 & 33 & 40 & 48 & 56 & 64 & 72 & 80 & 89 \\
\hline 29 & 18 & 26 & 33 & 41 & 49 & 57 & 65 & 73 & 82 & 90 \\
\hline 30 & 19 & 26 & 34 & 42 & 50 & 58 & 66 & 75 & 83 & 92 \\
\hline 31 & 19 & 27 & 34 & 42 & 51 & 59 & 67 & 76 & 85 & $9 ?$ \\
\hline 32 & 19 & 27 & 35 & 43 & 51 & 60 & 68 & 77 & 85 & 95 \\
\hline 33 & 20 & 27 & 36 & 44 & 52 & 61 & 70 & 78 & 87 & 96 \\
\hline 34 & 20 & 28 & 36 & 44 & 53 & 62 & 71 & 79 & 89 & 98 \\
\hline 35 & 20 & 28 & 37 & 45 & 54 & 63 & 72 & 81 & 90 & 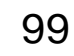 \\
\hline
\end{tabular}




$\begin{array}{lllllllllll}36 & 20 & 29 & 37 & 46 & 55 & 63 & 73 & 82 & 91 & 100 \\ 37 & 21 & 29 & 38 & 46 & 55 & 64 & 74 & 83 & 92 & 102 \\ 38 & 21 & 29 & 38 & 47 & 56 & 65 & 75 & 84 & 94 & 103 \\ 39 & 21 & 30 & 39 & 48 & 57 & 66 & 76 & 85 & 95 & 105 \\ 40 & 21 & 30 & 39 & 48 & 57 & 67 & 76 & 86 & 96 & 106 \\ 41 & 22 & 31 & 40 & 49 & 58 & 68 & 77 & 87 & 97 & 107 \\ 42 & 22 & 31 & 40 & 49 & 59 & 69 & 78 & 89 & 98 & 109 \\ 43 & 22 & 31 & 41 & 50 & 60 & 69 & 79 & 89 & 99 & 110 \\ 44 & 22 & 32 & 41 & 51 & 60 & 70 & 80 & 90 & 101 & 111 \\ 45 & 23 & 32 & 41 & 51 & 61 & 71 & 81 & 91 & 102 & 112 \\ 46 & 23 & 32 & 42 & 52 & 62 & 72 & 82 & 92 & 103 & 114 \\ 47 & 23 & 33 & 42 & 52 & 62 & 72 & 83 & 93 & 104 & 115 \\ 48 & 23 & 33 & 43 & 53 & 63 & 73 & 84 & 94 & 105 & 116 \\ 49 & 24 & 33 & 43 & 53 & 64 & 74 & 85 & 95 & 106 & 117 \\ 50 & 24 & 34 & 44 & 54 & 64 & 75 & 85 & 95 & 107 & 118 \\ 55 & 25 & 35 & 46 & 56 & 67 & 78 & 90 & 101 & 112 & 124 \\ 60 & 26 & 37 & 48 & 59 & 70 & 82 & 94 & 105 & 117 & 130 \\ 65 & 27 & 38 & 50 & 61 & 73 & 85 & 97 & 110 & 122 & 135 \\ 70 & 28 & 40 & 52 & 64 & 76 & 88 & 101 & 114 & 127 & 140 \\ 75 & 29 & 41 & 53 & 66 & 79 & 91 & 105 & 118 & 131 & 145 \\ 80 & 30 & 42 & 55 & 68 & 81 & 94 & 108 & 122 & 136 & 150 \\ 85 & 31 & 44 & 57 & 70 & 84 & 97 & 111 & 125 & 140 & 154 \\ 90 & 32 & 45 & 58 & 72 & 86 & 100 & 114 & 129 & 144 & 159 \\ 100 & 34 & 47 & 61 & 76 & 91 & 105 & 121 & 136 & 151 & 167\end{array}$

Fonte: ABNT - NBR 13170, 1994.

Anexo 4. Tabela com os valores críticos para comparação com os módulos das diferenças entre as somas das ordens do teste de ordenação, a $1 \%$ de significância

\begin{tabular}{|c|c|c|c|c|c|c|c|c|c|c|}
\hline \multirow{2}{*}{$\begin{array}{c}\text { № de } \\
\text { julgamentos }\end{array}$} & \multicolumn{10}{|c|}{$\mathrm{n}^{0}$ de amostras ou tratamentos } \\
\hline & 3 & 4 & 5 & 6 & 7 & 8 & 9 & 10 & 11 & 12 \\
\hline 5 & 9 & 13 & 16 & 19 & 23 & 26 & 30 & 33 & 37 & $\overline{41}$ \\
\hline 6 & 10 & 14 & 18 & 21 & 25 & 29 & 33 & 37 & 41 & 45 \\
\hline 7 & 11 & 15 & 19 & 23 & 28 & 32 & 36 & 40 & 45 & 49 \\
\hline 8 & 12 & 16 & 21 & 25 & 30 & 34 & 39 & 43 & 48 & 53 \\
\hline 9 & 13 & 17 & 22 & 27 & 32 & 36 & 41 & 46 & 51 & 56 \\
\hline 10 & 13 & 18 & 23 & 28 & 33 & 38 & 44 & 49 & 54 & 59 \\
\hline 11 & 14 & 19 & 24 & 30 & 35 & 40 & 46 & 51 & 57 & 63 \\
\hline 12 & 15 & 20 & 26 & 31 & 37 & 42 & 48 & 54 & 60 & 66 \\
\hline 13 & 15 & 21 & 27 & 32 & 38 & 44 & 50 & 56 & 62 & 68 \\
\hline 14 & 16 & 22 & 28 & 34 & 40 & 46 & 52 & 58 & 65 & 71 \\
\hline 15 & 16 & 22 & 28 & 35 & 41 & 48 & 54 & 60 & 67 & 74 \\
\hline
\end{tabular}




$\begin{array}{lllllllllll}16 & 17 & 23 & 30 & 36 & 43 & 49 & 56 & 63 & 70 & 77 \\ 17 & 17 & 24 & 31 & 37 & 44 & 51 & 58 & 65 & 72 & 79 \\ 18 & 18 & 25 & 31 & 38 & 45 & 52 & 60 & 67 & 74 & 81 \\ 19 & 18 & 25 & 32 & 39 & 46 & 54 & 61 & 69 & 76 & 84 \\ 20 & 19 & 26 & 33 & 40 & 48 & 55 & 63 & 70 & 78 & 86 \\ 21 & 19 & 27 & 34 & 41 & 49 & 56 & 64 & 72 & 80 & 88 \\ 22 & 20 & 27 & 35 & 42 & 50 & 58 & 66 & 74 & 82 & 90 \\ 23 & 20 & 28 & 35 & 43 & 51 & 59 & 67 & 75 & 84 & 92 \\ 24 & 21 & 28 & 36 & 44 & 52 & 60 & 69 & 77 & 85 & 94 \\ 25 & 21 & 29 & 37 & 45 & 53 & 62 & 70 & 79 & 87 & 96 \\ 26 & 22 & 29 & 38 & 46 & 54 & 63 & 71 & 80 & 89 & 98 \\ 27 & 22 & 30 & 38 & 47 & 55 & 64 & 73 & 82 & 91 & 100 \\ 28 & 22 & 31 & 39 & 48 & 56 & 65 & 74 & 83 & 92 & 101 \\ 29 & 23 & 31 & 40 & 48 & 57 & 66 & 75 & 85 & 94 & 103 \\ 30 & 23 & 32 & 40 & 49 & 58 & 67 & 77 & 86 & 95 & 105 \\ 31 & 23 & 32 & 41 & 50 & 59 & 69 & 78 & 87 & 97 & 107 \\ 32 & 24 & 33 & 42 & 51 & 60 & 70 & 79 & 89 & 99 & 108 \\ 33 & 24 & 33 & 42 & 52 & 61 & 71 & 80 & 90 & 100 & 110 \\ 34 & 25 & 34 & 43 & 52 & 62 & 72 & 82 & 92 & 102 & 112 \\ 35 & 25 & 34 & 44 & 53 & 63 & 73 & 83 & 93 & 103 & 113 \\ 36 & 25 & 35 & 44 & 54 & 64 & 74 & 84 & 94 & 105 & 115 \\ 37 & 26 & 35 & 45 & 55 & 65 & 75 & 85 & 95 & 106 & 117 \\ 38 & 26 & 36 & 45 & 55 & 66 & 76 & 86 & 97 & 107 & 118 \\ 39 & 26 & 36 & 46 & 56 & 66 & 77 & 87 & 98 & 109 & 120 \\ 40 & 27 & 36 & 47 & 57 & 67 & 78 & 88 & 99 & 110 & 121 \\ 41 & 27 & 37 & 47 & 57 & 68 & 79 & 90 & 100 & 112 & 123 \\ 42 & 27 & 37 & 48 & 58 & 69 & 80 & 91 & 102 & 113 & 124 \\ 43 & 28 & 38 & 48 & 59 & 70 & 81 & 92 & 103 & 114 & 126 \\ 44 & 28 & 38 & 49 & 60 & 70 & 82 & 93 & 104 & 115 & 127 \\ 45 & 28 & 39 & 49 & 60 & 71 & 82 & 94 & 105 & 117 & 128 \\ 46 & 28 & 39 & 50 & 61 & 72 & 83 & 95 & 106 & 118 & 130 \\ 48 & 29 & 40 & 51 & 62 & 74 & 85 & 97 & 109 & 121 & 133 \\ 50 & 30 & 41 & 52 & 63 & 75 & 87 & 99 & 111 & 123 & 135 \\ 60 & 32 & 45 & 57 & 60 & 82 & 95 & 108 & 121 & 135 & 148 \\ 70 & 35 & 48 & 61 & 75 & 89 & 103 & 117 & 131 & 146 & 160 \\ 80 & 37 & 51 & 66 & 80 & 95 & 110 & 125 & 140 & 156 & 171 \\ 100 & 42 & 57 & 73 & 89 & 106 & 123 & 140 & 157 & 174 & 191\end{array}$

Fonte: ABNT, NBR 13170, 1994 
Anexo 5. Tabela com os valores críticos do qui-quadrado.

\begin{tabular}{l|c|c|c|c|c|c}
\hline \multicolumn{7}{c}{ Níveis de significância } \\
\hline monótese & $0,10 \%$ & $0,05 \%$ & $\begin{array}{c}0,025 \\
\%\end{array}$ & $\begin{array}{c}0,01 \\
\%\end{array}$ & $\begin{array}{c}0,005 \\
\%\end{array}$ \\
\hline \multicolumn{1}{l}{ Grau de liberdade } & 1 & 2,71 & 3,84 & 5,02 & 6,63 & 7,88 \\
& 2 & 4,61 & 5,99 & 7,38 & 9,21 & 10,6 \\
& 3 & 6,25 & 7,81 & 9,35 & 11,3 & 12,6 \\
& 4 & 7,78 & 9,49 & 11,1 & 13,3 & 14,9 \\
& 5 & 9,24 & 11,1 & 12,8 & 15,1 & 16,7 \\
& 6 & 10,6 & 12,6 & 14,4 & 16,8 & 18,5 \\
& 7 & 12,0 & 14,1 & 16,0 & 18,5 & 20,3 \\
& 8 & 13,4 & 15,5 & 17,5 & 20,1 & 22,0 \\
& 9 & 14,7 & 16,9 & 19,0 & 21,7 & 23,8 \\
& 10 & 16,0 & 18,3 & 20,5 & 23,2 & 25,2 \\
\hline
\end{tabular}

Fonte: ABNT - NBR 13171 (1994). 


\section{Autores}

Cristina Jansen Alves ${ }^{1, *}$, Deborah Murowaniecki Otero ${ }^{2}$, Fernanda Doring Krumreich $^{1}$

1. Departamento de Ciência e Tecnologia de Alimentos, Universidade Federal de Pelotas, Capão do Leão, Brasil.

2. Departamento de Ciência dos Alimentos, Universidade Federal da Bahia, Campus Canela, 40110-907, Salvador, Brasil.

*Autor para correspondência: cris-jansen@hotmail.com 